\title{
Adaptive Dly-ACK for TCP over 802.15.3 WPAN
}

\author{
Hongyuan Chen*, Zihua Guo ${ }^{\ddagger}$, Richard $\mathrm{Yao}^{\ddagger}$, Yanda $\mathrm{Li}^{*}$ \\ * Department of Automation, \\ Tsinghua University, Beijing 100084, P.R. China \\ Email: chenhongyuan00@mails.tsinghua.edu.cn, daulyd@mail.tsinghua.edu.cn \\ $\ddagger$ Microsoft Research, Asia \\ 3F, Beijing Sigma Center, No. 49, Zhichun Road \\ Haidian District, Beijing 100080, P. R. China \\ Email: \{zhguo, richyao\}@microsoft.com
}

\begin{abstract}
Dly-ACK scheme in IEEE 802.15.3 is designed to reduce the overhead of the ACK and improve the channel utilization. However, how to use the Dly-ACK is open for implementation. In this paper, we first investigate the problems of applying fixed Dly-ACK scheme to the TCP stream and show that the TCP performance is rather poor. Then, we propose two enhancement mechanisms for TCP with Dly-ACK over 802.15.3 system. The first one is to request the Dly-ACK frame adaptively or change the burst size of Dly-ACK according to the queue size. The second is a retransmission counter to enable the destination DEV to deliver the packets to upper layer timely and orderly. Simulation results show that, with our enhancements, the TCP throughput can be improved more than $40 \%$ compared with the conventional Imm-ACK. We also investigate the impacts of some important system parameters such as the buffer size on the TCP performance. Some guidelines for the Dly-ACK design are given. Finally, it is worth to point out that our enhancements are compatible with the standard.
\end{abstract}

\section{INTRODUCTION}

IEEE 802.15.3 Wireless Personal Area Network (WPAN) [1], [2] is designed for short-range ad hoc connectivity among portable devices. It has gained much attention in industry recently. The standard defines the physical layer (PHY) and the medium access control (MAC) layer. The network formed by 802.15 .3 is called a piconet which allows a number of independent devices (DEVs) to communicate with each other in a peer-to-peer mode. The basic component of a piconet is the DEV. One of the DEVs is selected to serve as the piconet coordinator (PNC). The PNC provides some centralized controlling functionalities for the piconet.

Currently, the maximum data rate defined in 802.15.3 is $55 \mathrm{Mbps}$. The industry is defining the Ultra-Wideband (UWB) technology [3] as an alternative 802.15.3 PHY. The UWB technology is able to provide a data rate up to $480 \mathrm{Mbps}$. Because the wireless channel is error-prone, the MAC layer always adopts ACK and retransmission mechanism to provide reliable transmission for upper layer. However, in high data rate WPAN system, e.g., with UWB, the overhead caused by ACK may consume significant bandwidth. Table I in Section II-A presents the ACK overhead significance with an UWB PHY. To solve this problem, a new ACK policy, delayed acknowledgement (Dly-ACK), has been proposed in 802.15.3. In the Dly-ACK mechanism, instead of acknowledging each data frame, a burst of data frames is received, and then the whole burst is acknowledged by one ACK frame. Although the 802.15.3 standard defines the Dly-ACK scheme, how to use the Dly-ACK is open for implementation. In particular, as a MAC layer technology, it is necessary to study the Dly-ACK behavior when working with TCP. In this paper, we will show that Dly-ACK scheme with fixed burst size is unable to work well with the TCP and the TCP performance is rather poor. Therefore, we propose some enhancements for fixed Dly-ACK over TCP. Simulation results show that with our proposed enhancements, the ACK overhead is greatly reduced and TCP performance can be significantly improved.

The rest of this paper is organized as follows. In Section II, the 802.15.3 WPAN system and the ACK policies are briefly introduced. In Section III, the problem of fixed Dly-ACK for TCP and our proposed Dly-ACK enhancements are presented. Performance of our proposed Dly-ACK enhancements is given in Section IV, and finally the conclusions are drawn in Section $\mathrm{V}$.

\section{OVERVIEW OF IEEE 802.15.3}

In the MAC of 802.15.3, the time is divided into multiple superframes. The superframe is composed of three parts:

1) The beacon: Broadcast the time slot information for each $\mathrm{DEV}$ and management information for the piconet.

2) The optional contention access period (CAP): Communicate commands and/or asynchronous data based on CSMA/CA protocol.

3) The channel time allocation period (CTAP): It is composed of channel time allocations (CTAs), which are used for commands, isochronous streams and asynchronous data connections based on TDMA protocol.

The 802.15.3 MAC defines three types of ACK policies, i.e., no ACK (No-ACK), immediate ACK (Imm-ACK) and delayed ACK (Dly-ACK). When using the No-ACK policy, the destination DEV shall not acknowledge the received frame. The two successive frames are separated by minimum interframe space (MIFS). The No-ACK policy is appropriate for frames that do not require guaranteed delivery. The Imm-ACK policy provides an ACK process in which each data frame is individually ACKed following the reception of the frame. All frames, including the data frames and the ACK frame, are separated by the short interframe space (SIFS, larger than 


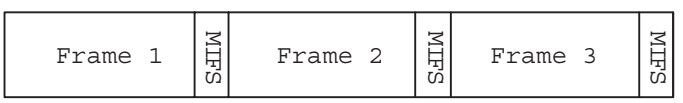

(a) No-ACK

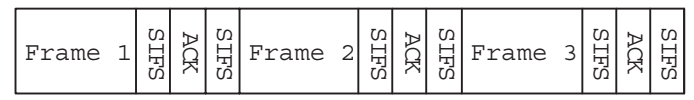

(b) Imm-ACK

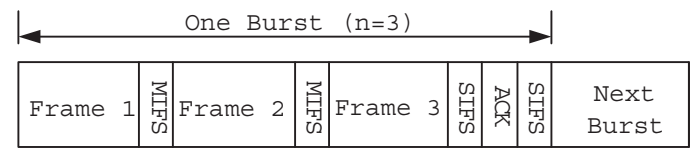

(c) Dly-ACK

\begin{tabular}{|c|c|c|c|c|c|c|c|}
\hline bytes $: 4$ & 2 & $\cdots$ & 2 & 1 & 1 & 1 & 10 \\
\hline FCS & $\begin{array}{l}\text { MPDU ID } \\
\text { block-n }\end{array}$ & $\ldots$ & $\begin{array}{l}\text { MPDU ID } \\
\text { block-1 }\end{array}$ & $\begin{array}{c}\text { MPDUs } \\
\text { ACKed }\end{array}$ & $\begin{array}{c}\text { Max } \\
\text { frames }\end{array}$ & $\begin{array}{c}\text { Max } \\
\text { burst }\end{array}$ & $\begin{array}{c}\text { MAC } \\
\text { header }\end{array}$ \\
\hline
\end{tabular}

(d) Dly-ACK frame format

Fig. 1. ACK policies in the IEEE 802.15.3

MIFS). The Dly-ACK policy allows the source DEV to send a burst of frames without the intervening ACK frames. When necessary, the source adds Dly-ACK request information to a frame's MAC header. Once the destination receives this frame which includes request information, it will send the Dly-ACK frame which acknowledges those correctly received frames in current burst. The source shall not start or resume the next burst transmission until a Dly-ACK frame is received. These frames which are not ACKed should be retransmitted in the next burst. The three ACK policies are shown in Fig. 1 and Fig.1(d) gives the Dly-ACK frame format. The Max Burst filed indicates the number of frames with the maximum size that may be sent in one burst. The Max Frames field denotes the maximum number of frames regardless of size that may be sent in one burst.

In this paper, we call the number of frames that are transmitted in a burst as burst-size $n$. For convenience, we use $n-D l y-A C K$ to represent the Dly-ACK mechanism which adopts $n$ as its burst-size. Obviously, the Imm-ACK and No$\mathrm{ACK}$ are special cases of Dly-ACK with $n$ being 1 and infinite, respectively. The No-ACK policy does not have any influence on the channel utilization, but it is not suitable for the reliable transmission applications, which usually adopt TCP as the transport protocol. Because the wireless channel is error-prone, it is necessary to provide a reliable wireless link for the TCP [4]. Thus, the Dly-ACK or Imm-ACK is needed.

From Fig.1, we can compute the ACK overhead under various data rates. When the Imm-ACK is used, the average total transmission time $t_{m}$ of one frame is $t_{p}+t_{A C K}+2 t_{S I F S}$ with $t_{p}, t_{A C K}, t_{S I F S}$, denoting the length of the data packet, the ACK packet and the SIFS, respectively. When the n-DlyACK is used, $t_{m}$ is $\left(n t_{p}+t_{A C K}+(n-1) t_{M I F S}+2 t_{S I F S}\right) / n$. Now, we assume the basic data rate is $100 \mathrm{Mbps}$, the frame size is 1000 bytes. The other parameters, e.g., MAC header, PHY header, etc., are according to [1]. In Table I, we list the values
TABLE I

TRANSMISSION TIME UNDER VARIOUS DATA RATES

\begin{tabular}{|c|c|c|c|c|c|c|}
\hline \multirow{2}{*}{$\begin{array}{c}\text { Data rate } \\
(\mathrm{Mbps})\end{array}$} & \multicolumn{2}{|c|}{ Imm-ACK } & \multicolumn{2}{c|}{ 5-Dly-ACK } & \multicolumn{2}{c|}{ 10-Dly-ACK } \\
\cline { 2 - 7 } & $t_{m}(\mu s)$ & $t_{p} / t_{m}(\%)$ & $t_{m}$ & $t_{p} / t_{m}$ & $t_{m}$ & $t_{p} / t_{m}$ \\
\hline 100 & 120 & 75.0 & 97.9 & 91.9 & 95.0 & 94.7 \\
\hline 200 & 80 & 62.5 & 57.9 & 86.4 & 55.0 & 90.9 \\
\hline 480 & 56.7 & 47.1 & 34.5 & 77.2 & 31.7 & 81.6 \\
\hline
\end{tabular}

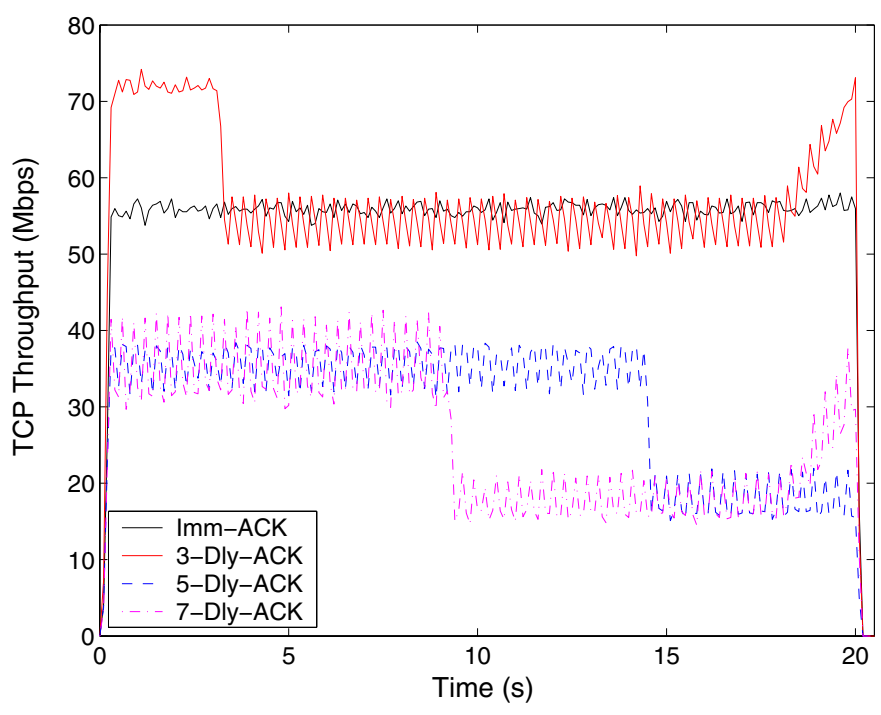

Fig. 2. Problem of fixed Dly-ACK for TCP

of $t_{m}$ under various data rates and the ratio of $t_{p} / t_{m}$ without channel error. We can see that under high data rate for Imm$\mathrm{ACK}$, among the total time required to successfully transmit a MAC frame, only a small portion of the time is really used to send the payload. Therefore, we can see that the Dly-ACK policy gives much room to improve the channel utilization.

\section{AdAPTIVE Dly-ACK FOR TCP}

\section{A. Problem of fixed Dly-ACK}

In [5], it is pointed out that the throughput of a bulk TCP is a monolithically decreasing function of the round trip time (RTT). That is, the smaller RTT, the larger throughput. For RTT of a TCP packet in 802.15.3 system, the transmission time $t_{m}$ in the MAC layer is an important element. According to Table I, the Dly-ACK policy may be a good choice to enhance the TCP performance by reducing RTT and improve the channel utilization. However, if we use fixed burst size nDly-ACK, there are some serious problems over an erroneous wireless channel. In Fig.2, we presented the TCP performance with Dly-ACK for $n=1,3,5$ and 7 over a channel with Frame Error Rate $(\mathrm{FER})=20 \%$. From Fig.2, we can see that only Imm-ACK $(n=1)$ works well. In the following, we will present the problems of fixed Dly-ACK in details.

1) The local information problem: According to 802.15 .3 specification, the MAC frames should be delivered to upper layer in order. Thus, some frames with higher MAC-id correctly received must wait for the missing frames, which 
have lower MAC-id and are wrong due to channel errors. In general, each MAC frame has the retransmission limit $r_{l}$. If one frame's retransmission limit has been reached, the source will discard this frame. Then, it is unnecessary for those waiting frames in the destination to wait for this dropped frame any more. However, the destination DEV cannot know that the missing frame has been dropped by the source DEV. Thus, those waiting frames may still wait and cannot be delivered to upper layer timely. This finally will result in a time-out event at TCP and degrade performance significantly. Therefore, a mechanism is needed to solve this problem. For some delay-sensitive applications, this problem can be solved by adding a delay timer. But it is inappropriate for TCP. If the timer is set too short, it may result in unnecessary retransmission at TCP because the missing frame may be received correctly in the next retransmission. On the other hand, if the timer is set too long, it may cause a time-out at TCP. Here we propose a retransmission counter (Re-Counter) in the destination DEV to overcome this problem. That is, once a frame is received in error, the destination DEV will begin to count the retransmission time which is actually the number of Dly-ACK frame that acknowledges the incorrect reception of this frame. Once the Re-counter exceeds the limit $r_{l}$, those waiting frames no longer wait for this missing frame and should be delivered to upper layer immediately.

2) The fixed burst-size problem: The TCP packet transmission depends on the congestion window. Thus, sometimes the MAC traffic queue will be empty. In this case, when using DlyACK policy whose burst-size $n$ is fixed, there are two negative effects on the performance. First, it will cause some additional delay for the source MAC to wait for the ACK frame. Second, the time-out event will occur at TCP layer. We use an example to explain these two problems. Assuming that the burst-size is constant and $n=10$. At time $t$, the congestion window at TCP is 16 and there are 16 outgoing packets in source MAC. At this time, the TCP will pass nothing to the MAC layer until it receivers some TCP layer acknowledgements for the 16 packets. Let us see what happen at MAC layer. The 16 packets will be transmitted in two bursts. In the first burst, packets 1-10 are transmitted. Then, there are two possibilities. The first case is that there is no error in this burst. Then, packets 11-20 will be passed in the second burst. Note that there are no any packet in source buffer after packet 16 is transmitted. Moreover, after packets 11-16 have been transmitted, the source TCP just receives ACK frames of packets 1-10 and injects packets 1720 into MAC layer. Only when packets $11-20$ are received at destination MAC, the Dly-ACK frame will be sent to source MAC. Therefore, for packets 11-16, they need longer time to obtain Dly-ACK frame than packets 1-10. The second case is that there are some errors in the first burst. For example, packet 1 is in error. Then, the destination MAC does not pass any packets to upper layer due to missing packet 1 , even if packets 2-10 are correctly received. Now, in the source side, packets 17-20 cannot be injected to MAC layer because no any TCP ACK packets are received at source TCP. Then, the source will send packets 1,11-16 in the second burst.
Unfortunately, packet 1 is in error again. Then, after packet 16 is transmitted, the source MAC will wait packet $17-19$ to fill this burst. However, the destination MAC does not generate the Dly-ACK frame because it has only received seven packets for the second burst. Furthermore, since packet 1 is in error, no packet is delivered to TCP layer and thus, no TCP ACK will be sent to the source. Then, there is a deadlock between the source and the destination. For the source, it needs some TCP layer ACK to generate more packets so that the second burst is filled to enable the destination to generate a Dly-ACK frame at MAC. For the destination, packets cannot be delivered. This will be solved until a time-out is triggered at source TCP.

If the source MAC requests the Dly-ACK frame when it transmits packet 16, these two problems can be solved efficiently. First, it reduces the additional wait time for the packets 17-20. Second, the deadlock will not occur between the source and destination. Therefore, we propose that the source MAC should request the Dly-ACK frame once it detects its buffer is empty.

\section{B. Proposed Enhancement for TCP over fixed Dly-ACK}

According to the above discussion regarding the interaction between TCP and fixed Dly-ACK, we propose the following mechanisms to enhance the fixed Dly-ACK for TCP.

At source DEV's MAC:

1) Before sending a packet, it detects the traffic buffer. If the buffer is empty, it should add a Dly-ACK request information to the MAC header of the frame which is going to be transmitted. Else, go to 2).

2) It sends frames by means of $k$-Dly-ACK policy. The parameter $k$ is determined by $\min \left(n, b_{l}\right)$ where $n$ is an integer and is within [5,10] and $b_{l}$ is the destination's remaining buffer size and is obtained from the latest Dly-ACK frame sent from the destination DEV. If the destination DEV's buffer is large enough, the source DEV will request a Dly-ACK regularly for every $n$ frames, which is an n-Dly-ACK. We will show the effect of $n$ on the TCP performance in Section IV.

At destination DEV's MAC:

1) Deliver correctly received frames to upper layer in order.

2) If one frame is missing due to channel error, these correctly received frames with higher MAC-id should always wait for the missing frame before its Re-Counter reaches the retransmission limit $r_{l}$.

3) Once the missing frame's Re-Counter exceeds $r_{l}$, the other frames in the buffer waiting for this frame should be delivered to upper layer according to their MAC-id order.

4) When receiving the Dly-ACK requesting information, the destination responds a Dly-ACK frame by setting the max burst in the MAC header to be the remaining buffer size.

\section{Simulation Results}

To show potential of our proposed mechanism, we present some simulation results obtained by $n s-2$ [6] here. One TCP connection is established between the PNC and one DEV in 
TABLE II

SOME System PARAMETERS USED TO ObTAIN RESUlTS

\begin{tabular}{ll}
\hline Packet length & 1000 bytes \\
MAC head & 10 bytes \\
PHY head & $9.4 \mu \mathrm{s}$ \\
Imm-ACK frame length & MAC head + PHY head \\
n-Dly-ACK frame length & MAC head + PHY head $+(2 \mathrm{n}+7)$ bytes \\
Basic Data Rate & 100 Mbps \\
Channel rate & $200 \mathrm{Mbps}$ \\
Super frame size & $15 \mathrm{~ms}$ \\
Retransmission limit $r_{l}$ & 5 \\
MIFS & $2 \mu \mathrm{s}$ \\
SIFS & $10 \mu \mathrm{s}$ \\
\hline
\end{tabular}

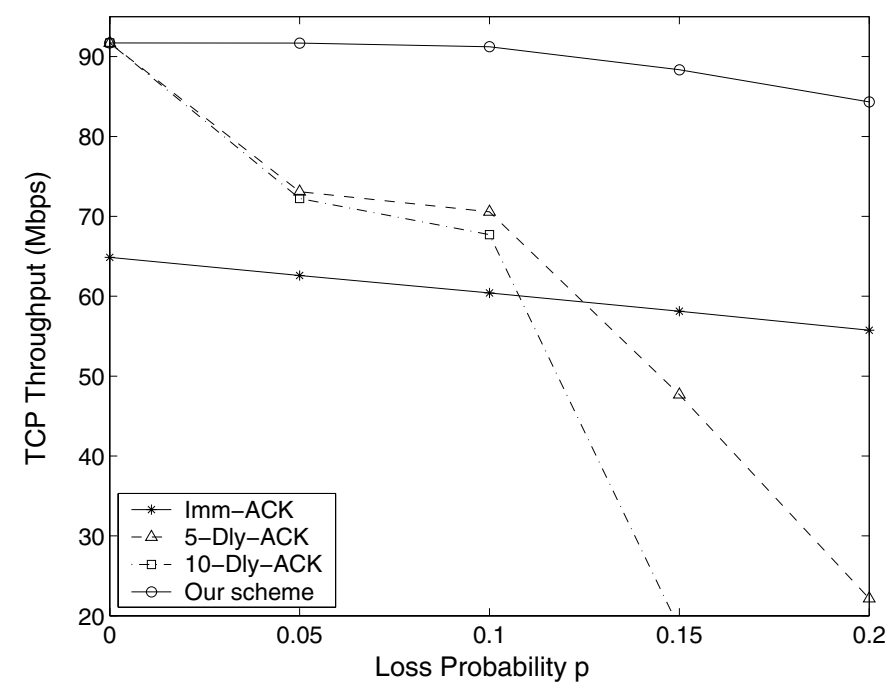

Fig. 3. Performance comparison

the piconet. We assume that each TCP connection carries a bulk file transfer. Because the CAP period is optional, we only consider the CTAP period. Assuming that frame loss follows a Bernoulli model, i.e., each frame's loss is independent and with a probability $p$, which is the FER. Some important parameters are listed in Table II and others are determined according to the standard [1].

First, we compare the performance of our enhanced adaptive Dly-ACK with Imm-ACK and fixed Dly-ACK. Fig.3 illustrates the aggregate throughput at TCP under various $p$. In these simulations, TCP Reno is used and the destination's MAC buffer size is $400 \mathrm{kbits}$ (correspond to 50 frames). The parameter $n$ is set to 10 . We can see that in all cases, the throughput of our proposed enhanced Dly-ACK is much larger than that of the Imm-ACK. Fig. 3 indicates that the throughput gain is more than $40 \%$ usually. For the fixed 5-Dly-ACK and 10-Dly-ACK, although their performances are good at low FER, however, they degrade quickly when the FER increases.

The throughput gain is mainly due to the decrease of RTT as well as the increase of the channel utilization. The TCP packets' RTT of one flow are shown in Fig.4 with $p=0.1$. From this figure, it is clear that the RTT can be greatly reduced by our scheme. Thus, the throughput can be increased significantly.

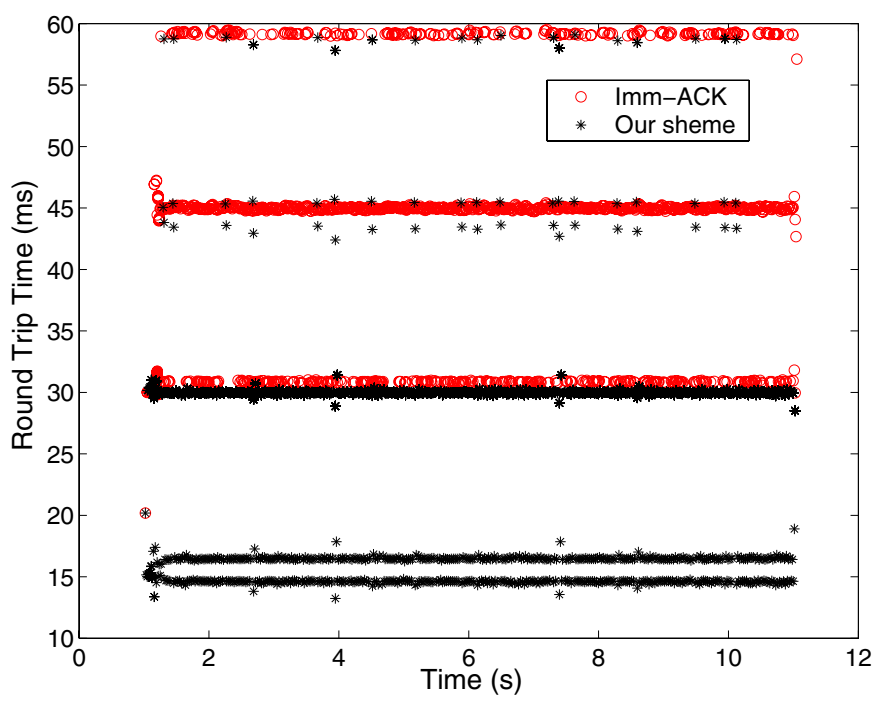

Fig. 4. RTT over time, $p=0.1, n=10$

Next, let us discuss the impact of some parameters of the Dly-ACK on TCP performance. As mentioned before, the source sends a Dly-ACK request after $k$ frames with $k=\min \left(n, b_{l}\right)$. Obviously, the parameter $n$ should play an important role on the performance. The results of throughput vs. $\mathrm{n}$ are shown in Fig.5(a). In this figure, to show the effect of $n$ more clearly, the destination's buffer is set to be large enough to accommodate all correctly received frames. We list the results when $p=0.05$ and 0.1 , respectively. We can see that in the two scenarios, the throughput becomes larger with the increasing of $n$ when $n$ is lower than 8 . However, when $n$ is larger than 8 , the throughput is almost stable. Although the performance may become a little better when $n$ is larger than 10 under $p=0.05$ or 0.1 , a much larger buffer is required in the destination MAC. In Fig.5(b), we present the number of frame in the destination's buffer with $n=10$ and 20, respectively under $p=0.1$. From this figure, we can see that the queue length is seldom larger than 20 when $n=10$. However, when $n=20$, the peak is as large as 50 . Thus, our suggestion is that $n$ may be around 5 10 and larger value is unnecessary.

Finally, we are to investigate the effect of the destination's buffer size on the TCP performance. As mentioned above, the larger $n$, the larger buffer size is required to fully accommodate the received frame before they are delivered to the upper layer. However, it is interesting to see, for a given $n$, how much the buffer size affects the performance. Fig. 6 shows the TCP throughput as a function of the buffer size under various loss probability $p$ with $n=10$. When the loss probability is low $(p=0.05)$, the system throughput is almost constant regardless the buffer size. This is easily explained because there are seldom MAC frames in error and the destination buffer is usually empty since each frame can be delivered from MAC layer to upper layer immediately. However, when there is a high loss probability, e.g., $p=0.2$, the buffer size has a notable impact on the performance. This is because we set the burst size $k=\min \left(n, b_{l}\right)$. As loss probability is high, there may 


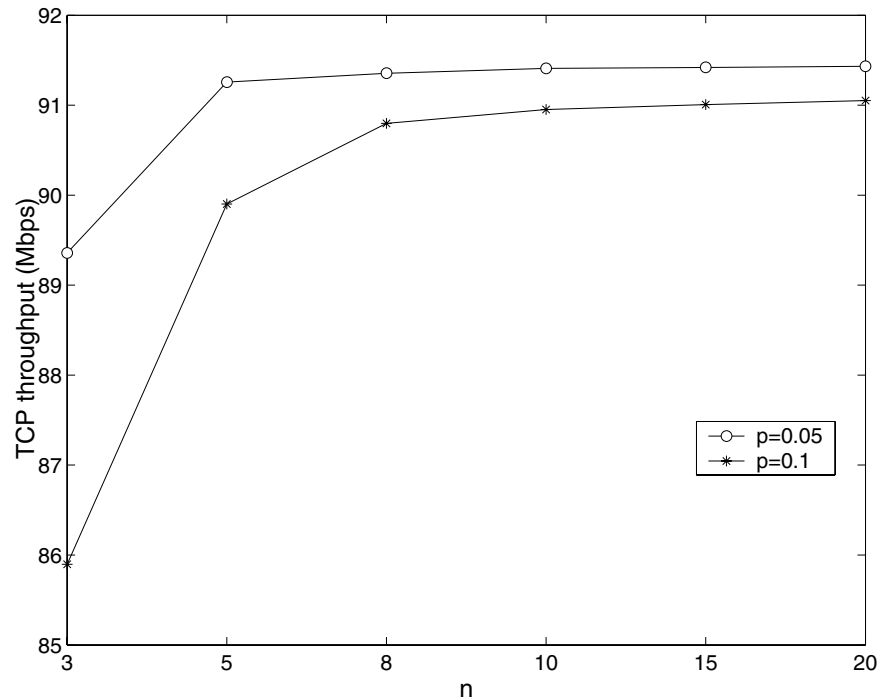

(a) Throughput vs. $n$

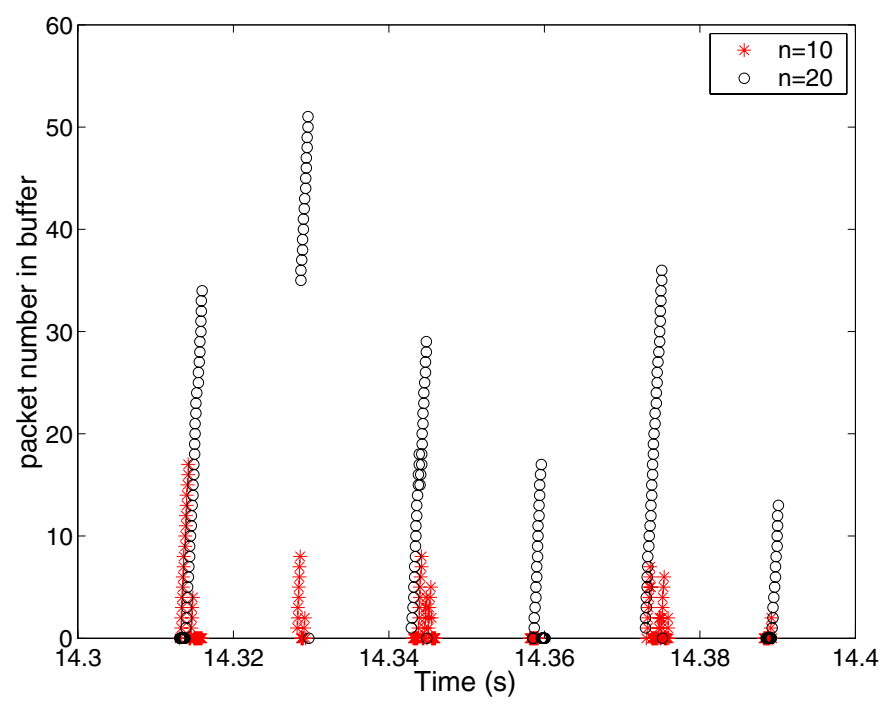

(b) Queue Length, $p=0.1$

Fig. 5. Effects of $n$

be a lot of frames in the buffer that cannot be delivered to upper layer timely. Thus, there is little remaining space to accommodate more upcoming frames. Finally, this results in the reduction of $k$. As a result, the performance degrades. To gain further insight about this problem, we list the distribution of burst size $k$ in Table III. These results are collected from a simulation which runs within a duration of 20 seconds under high loss probability. Obviously, $k$ has a higher average value when the buffer size is larger, which indicates a better channel utilization. Furthermore, the total number of transmission burst (i.e., the count of Dly-ACK frames) in the entire simulation duration becomes smaller with larger buffer size.

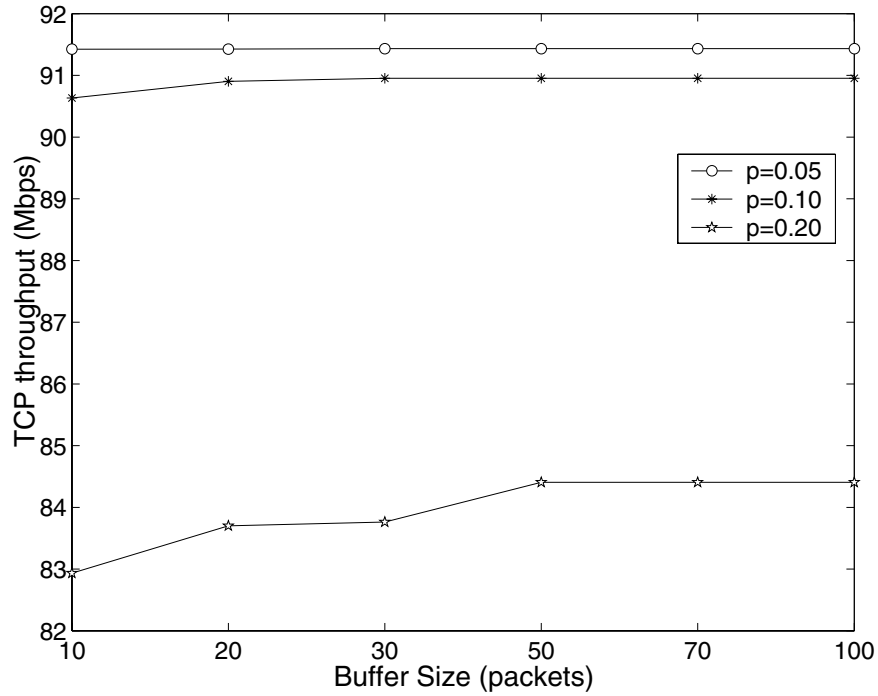

Fig. 6. Effects of buffer size, $n=10$

TABLE III

The Distribution of Burst Size $k(p=0.2, n=10)$

\begin{tabular}{|c|c|c|c|c|c|c|c|}
\hline buffer size & $\mathrm{k} \leq 5$ & 6 & 7 & 8 & 9 & 10 & Total \\
\hline 10 & 3128 & 504 & 470 & 451 & 454 & 2497 & 7504 \\
\hline 20 & 925 & 162 & 182 & 186 & 200 & 4651 & 6306 \\
\hline 100 & 655 & 65 & 98 & 93 & 72 & 5098 & 6081 \\
\hline
\end{tabular}

\section{CONCLUSIONS}

In this paper, we studied the performance of Dly-ACK for TCP in IEEE 802.15.3 WPAN system. It is observed that the fixed Dly-ACK does not give a satisfied performance for TCP due to some problems. Based on these observations, we proposed two enhancement schemes to improve the TCP performance with adaptive Dly-ACK burst size. The simulation results show that the TCP throughput with our enhanced DlyACK can be significantly improved compared with the ImmACK. The impacts of some Dly-ACK parameters on the TCP performance are also studied.

\section{REFERENCES}

[1] "Part 15.3: Wireless medium access control (MAC) and physical layer (PHY) specifications for high rate wireless personal area networks (WPAN)," IEEE Std 802.15.3, Sep 2003.

[2] K. Jeyhan and B. Corporation, "High-rate wireless personal area networks," IEEE Commun. Mag., pp. 96-102, Dec 2001.

[3] D. Porcino and W. Hirt, "Ultra-wideband radio technology: Potential and challenges ahead," IEEE Commun. Mag., pp. 66-74, Jul 2003.

[4] X. George, C. P. George, M. Petri, and S. Mika, "TCP performance issues over wireless links," IEEE Commun. Mag., pp. 52-58, Apr 2001.

[5] J. Padhye, V. Firoiu, F. Towsley, and J. F. Kurose, "Modeling TCP reno performance: A simple model and its empirical validation," IEEE/ACM Trans. Networking, vol. 8, no. 2, pp. 133-145, Apr 2000.

[6] "NS-2," http://www.isi.edu/nsnam/ns/. 